\title{
INSTITUCIONES INFORMALES, COALICIONES Y GABINETES EN EL PRESIDENCIALISMO CHILENO
}

\author{
María de los Ángeles Fernández Ramil (mangeles.fernandez@chile21.cl) \\ Eugenio Rivera Urrutia (eugenioriveraurrutia@gmail.com) \\ Fundación Chile 21
}

\begin{abstract}
El presente artículo busca contribuir al estudio del presidencialismo desde la óptica de la constitución de gabinetes y su operación en gobiernos de coalición, aportando una visión dinámica al enfoque de instituciones informales a partir del caso chileno. Mediante el análisis de los gobiernos desde 1990, se argumenta que la designación de los gabinetes resulta de interacciones complejas entre la autoridad presidencial, las organizaciones políticas formales y mecanismos informales influidos, tanto por historias comunes como por complicidades que expresan, en forma ambivalente, la solidez y deterioro de los partidos y de las alianzas políticas. Al mismo tiempo se sostiene que la relación entre el Presidente y sus gabinetes incide en la base política de sus respectivas administraciones, fortaleciéndola o deteriorándola.
\end{abstract}

Palabras clave: Presidencialismo, gabinetes, Chile.

\section{INFORMAL INSTITUTIONS, COALITIONS AND CABINETS: PRESIDENTIALISM IN CHILE}

This article seeks to contribute to the study of presidentialism from the standpoint of the constitution of cabinets and their operation in coalitiong o vernments. The article also provides a dynamic vision on approaches to informal institutions based on politics in Chile. Through a comparative analysis of governments since 1990, it becomes clear that the designation of cabinet members comes from a complex interaction between the presidential authority, formal political organizations, and informal mechanisms. All three blocs are influenced by common histories and complicities that expresses themselves, in an ambivalent form, in the strengthening and deterioration of political parties and political alliances. At the same time, the relationship between president sand their cabinets has had an impact on the political base of the irrespective administrations, either strengthening or weakening them.

Keywords: Presidentialism, cabinets, Chile. 


\section{Introducción}

Desde que se inaugurara la transición a la democracia en 1990, el presidencialismo chileno logró sortear los malos presagios que, a los fines de la gobernabilidad y estabilidad democráticas, parecen acompañar el funcionamiento de este tipo de regímenes para el caso latinoamericano ${ }^{1}$. No abstante, a partir del gobierno de Ricardo Lagos, aparecieron evidencias de las dificultades propias del presidencialismo identificadas por la literatura. Se observan, entre otros fenómenos, la falta de disciplina partidaria y la salida de parlamentarios de varios de los partidos de la Concertación de Partidos por la Democracia, coalición gobernante de centroizquierda, en el contexto de una progresiva deslegitimación de los partidos y del Congreso. Del mismo modo, la eficiente gestión gubernamental de los primeros años de la transición, que permitía hablar del caso chileno como un modelo a imitar, dio paso a una serie de dificultades como las que se enfrentaron, por ejemplo, con la crisis del gas a partir del 2004 o los problemas de la reforma del sistema de transporte urbano del Gran Santiago, en 2007. Problemas como los señalados, sumados a la movilización social que surge con fuerza a partir del 2011, comienzan a poner en cuestión el exitoso desempeño del "modelo" chileno. La paradoja es que, a la vez que la actividad política se enmarca en un sistema de partidos estable e institucionalizado, éste coexiste con débiles vínculos con la sociedad (Luna y Mardones, 2011: 203).

El caso chileno es considerado anómalo en el contexto latinoamericano (comparable solo con la estabilidad de Uruguay) ya que, a pesar de ser "quizás el país con la estructura institucional más problemática de América Latina” (Siavelis, 2000), llamaba la atención el éxito de su transición. Según Mainwaring y Shugart (2002: 265), ello se debía a "la naturaleza consocional de la transición del autoritarismo y la voluntad de los partidos de participar en listas conjuntas como resultado de un sistema electoral restrictivo", anticipando, además, que "era difícil que la baja fragmentación perdurase". Para Siavelis (2000: xvi) se explicaba por "factores contextuales de la transición misma como también por su efecto sobre el sistema de partidos, la estructura de incentivos y las decisiones de las élites gobernantes, que temperaban las orientaciones negativas asociadas a su presidencialismo exagerado y al sistema electoral mayoritario". Sin embargo, el autor anticipaba que "de no tener lugar pronto reformas institucionales y electorales, los conflictos intrapartido

Al respecto, referencia inexcusable es el libro de Mainwaring y Shugart (2002). 
e intrapoderes se evidenciarían, con problemas para la gobernabilidad democrática y limitaciones de su eficacia política".

Lo señalado plantea la necesidad de explicar los motivos de superación de los problemas del presidencialismo en una etapa para que luego, en la última década, se observen dificultades. Es por esto que el objetivo del presente artículo es, a partir de la historia transicional reciente de Chile, contribuir al conocimiento y análisis del funcionamiento del presidencialismo mediante la constitución de los gabinetes ministeriales y su operación en gobiernos de coalición. Utilizando el método "entrevista a élites", tomando como base una muestra de actores relevantes de las distintas administraciones y la revisión de la estructuración de los gabinetes ministeriales de los sucesivos gobiernos, se argumenta que su conformación resulta de interacciones complejas entre la autoridad presidencial, las organizaciones políticas formales e informales estructuradas sobre la base de historias comunes y complicidades que expresan, tanto la solidez como el deterioro de los partidos y de las alianzas políticas. Es ésta una dimensión relevante por cuanto la operación de la Presidencia y de sus gabinetes incide en la base política de las administraciones, fortaleciéndola o bien deteriorándola al punto que, si bien los gabinetes chilenos partieron, en 1990, siendo estructurados en base a criterios de representación partidistas formales, éstos fueron crecientemente sustituidos por la operación de instituciones informales. Se llama la atención, además, acerca de la tensión continua entre la toma de decisiones sobre la constitución y operación de los gabinetes y el relato que se construye sobre esas decisiones. Por medio de un análisis comparativo de los procesos de designación de los gabinetes ministeriales y de la toma de decisiones que caracteriza dicho proceso, centrado en los cinco gobiernos desde 1990 a la fecha, se enfatizan los denominados "mecanismos institucionales informales", entendidos como dispositivos que permiten adaptar los rígidos mecanismos formales a las realidades políticas, aun cuando en ocasiones sirven para encubrir el efecto de otros mecanismos (Gretchen y Levitsky, 2004). Desde esta perspectiva, la indagación enlaza con la tradición del institucionalismo histórico según la cual, "dentro del ámbito gubernamental, un programa o una organización toma determinado rumbo, hay una suerte de inercia que hace que las decisiones políticas iniciales persistan" (Peters, 2003: 99). En lo específico, incorpora el aporte conceptual realizado por Siavelis (2009) acerca del llamado "partido transversal”. Igualmente, se vincula con la investigación sobre la conformación de los ministerios en Chile en lo referido a los llamados estudios directos de los gabinetes (Avendaño y Dávila, 2012).

En la primera sección, se formulan algunas consideraciones conceptuales acerca de las limitaciones que, para el caso chileno, presentan enfoques anclados en las 
disposiciones institucionales de carácter formal y, al mismo tiempo, se sostiene que los éxitos en materia de estabilidad democrática en el contexto del régimen presidencial han sido posibles por la renuncia a los objetivos transformadores con que la Concertación asumió el gobierno. Enseguida, se realiza un análisis de la forma como se constituyeron los gabinetes iniciales en los períodos presidenciales analizados y la evolución del denominado "partido transversal". Luego se analiza el papel predominante del Ministro de Hacienda en el gobierno. Se concluye enfatizando la necesidad de profundizar la indagación de los mecanismos informales que subyacen a la lógica de funcionamiento del presidencialismo, reafirmando su importancia analítica en cuanto objeto de estudio en sí mismo.

\section{El presidencialismo y las limitaciones del formalismo institucional}

El análisis de la presidencia, de los equipos ministeriales y de sus relaciones con el Congreso ha sido una preocupación relevante de la investigación académica de los últimos años. Una primera línea se ha concentrado en indagar las dificultades que un sistema multipartidario plantea al presidencialismo para el impulso de su agenda política. Linz (1994) observaba la existencia de obstáculos para formar gobiernos de coalición en regímenes presidenciales como producto de los costos que implicaba para cualquier partido unirse a un gobierno presidencial. En un sentido similar argumentaban Mainwaring y Shugart (1997), subrayando los bajos incentivos existentes para que los partidos cooperen con el Presidente. No obstante, hasta principios de la primera década del siglo XXI, la Concertación logró superar estos condicionamientos y gobernar en condiciones auspiciosas. Frente a la tendencia a relevar las disposiciones institucionales formales que interactúan con el régimen presidencial tales como el número de partidos y su grado de fragmentación, fue emergiendo la idea de su insuficiencia para dar cuenta, tanto de éxitos como de problemas que encaraban este tipo de sistemas. Una contribución clave en esta materia es la de Helmke y Levitsky (2004) al señalar que "la atención cuidadosa a instituciones informales es crítica para entender los incentivos que habilitan y constriñen el comportamiento político. Los actores políticos responden a una mixtura de incentivos formales e informales y, en algunas instancias, los incentivos informales se imponen a los formales... la consideración de las reglas informales son con frecuencia críticas para explicar los resultados institucionales". Refiriéndose a Chile, sostienen que estas instituciones han limitado el poder presidencial. Helmke y Levitsky las definen como "reglas socialmente compartidas, generalmente no escritas, que son creadas, comunicadas y hechas cumplir fuera de los canales socialmente 
sancionados. El concepto, utilizado por otros autores respecto del caso de Chile (Walker, 2003; Siavelis, 2006), permitió reconocer e investigar fenómenos de gran relevancia para la operación del complejo sistema político heredado del régimen militar. Ideas como "cuoteo", "partido transversal" y "democracia de los acuerdos" fueron desarrolladas como dispositivos que permitían enfrentar los dilemas de la fórmula presidencial heredada.

Desde otra perspectiva, Díaz-Tendero (2006: 93 y 89) llamó la atención sobre ciertas características de la transición "pactada" y la instalación de la idea de "gradualismo secuencial". Formulada por Edgardo Boeninger", "suponía la necesidad de conciliación de opuestos y evitar la acumulación de conflictos, descartándose como métodos de presión la agitación social y la movilización callejera, apuntando a gobernar con eficacia y con sentido de largo plazo". Se sustentó en una suerte de "iluminismo técnico con legitimidad democrática", es decir, grupos de "técnicos" sobre los cuales ha descansado el diseño específico de la acción pública en Chile desde 1990 en adelante.

Ambas perspectivas son útiles pero deben ser precisadas. ¿Con qué criterio evaluar el éxito o fracaso del presidencialismo en el período post-Pinochet? Una posibilidad es una visión que privilegia la estabilidad política, el crecimiento económico alcanzado y el avance logrado en la reducción de la pobreza. La dificultad radica en que esos indicadores muestran en la segunda década de la transición un comportamiento sustancialmente menos exitoso. Son numerosos los analistas que sostienen que el país tuvo un crecimiento económico menor, el sistema político está afectado en su legitimidad y, más recientemente, crecen las críticas al modelo económico ${ }^{3}$. Cabe, en consecuencia, profundizar el análisis respecto de los factores que contribuyeron al éxito de este presidencialismo que luego parecen operar en sentido contrario. Una segunda perspectiva es la que asocia el éxito o el fracaso del presidencialismo al logro de los cambios económico-sociales que la coalición que derrotó al régimen militar esperaba alcanzar. Desde este punto de vista, los éxitos resultan sustancialmente menores.

Ministro de la Secretaría General de la Presidencia del gobierno de Patricio Aylwin (1990-1994).

Es el caso de Siavelis (2009:4) que señala:“A lo largo de la década de 1990 Chile ha sido sistemáticamente reconocido como un país que experimentó una transición democrática modelo y como una de las democracias más exitosas de América Latina". No obstante, el autor a renglón seguido sostiene: "Con el paso de los años, algo del brillo se ha apagado en relación al modelo chileno. La mala gestión del gobierno, la percepción de un aumento de la corrupción y la escalada de protestas durante la administración socialista de la Presidenta Michelle Bachelet han dado lugar a un aumento de las críticas a la Concertación, coalición que ha gobernado Chile desde el retorno a la democracia en 1990". 
El debate en torno al rol de las instituciones informales en el desempeño relativamente exitoso del sistema político chileno remitió a una lectura equívoca del rol jugado por éstas en los resultados evaluados positivamente de la política chilena. De hecho, éstos se ven interpelados por el regreso de la controversia en torno al malestar ${ }^{4}$ que los chilenos estarían experimentando. Desde el punto de vista de la eficacia de la propia Concertación, el predominio de una perspectiva formal, la escasa atención al proceso político efectivo y la perspectiva autocomplaciente respecto de su propia gestión contribuyó a ignorar las dificultades de ese presunto poderoso presidencialismo para impulsar efectivamente el proyecto de democratización profunda que había sustentado el programa original de dicha coalición política (Varas, 2012: 21). Si bien el Ejecutivo chileno dispone de importantes facultades en el proceso legislativo (Boeninger, 2007), lo cierto es que las restricciones provenientes del sistema binominal y los altos quórum requeridos en materias cruciales dan al poder legislativo una capacidad de obstrucción que dificulta las posibilidades de impulsar el programa de la coalición gobernante. El chileno es lo que Siavelis (2002) denomina "un presidencialismo exagerado con Presidentes moderados" y una amplia literatura recogida por Nolte (2003) da cuenta de los factores que lo explican.

Un elemento clave para entender la estabilidad democrática bajo los gobiernos de la Concertación fue la operación de lo que se denominó el "partido transversal" entendido, de acuerdo a Siavelis (2009: 14) como "el grupo informal integrado por políticos claves, en los primeros gobiernos democráticos, quienes se definen a sí mismos como "líderes de la Concertación" más que como líderes de sus partidos". Un segundo elemento fue "el acuerdo tácito donde el presidente puede negociar con poderosos actores económicos y líderes de la derecha para alcanzar soluciones consensuadas sobre temas de legislación más controversiales, antes que sean presentados al Congreso" (Siavelis, 2009: 14). En ese sentido, sostiene el mismo autor que, "el compromiso de los presidentes de dejar intacto el modelo económico tranquilizó a las poderosas élites económicas cuya reacción a un posible cambio en la política podría haber sido extraordinariamente desestabilizadora". En suma, este enclave fue central en el mantenimiento de la transición democrática. Sin embargo, la falta de voluntad de los gobiernos democráticos para poner en discusión los

4 Se ha intentado explicar las causas de la movilización estudiantil de 2011 y la adhesión ciudadana a la consigna levantada por él, sintetizada en "No al lucro en educación". Se observan dos líneas: aquellos que afirman que es necesario profundizar el modelo y que lo que subyace es una demanda por mayor inclusión en el mercado y otras que apuntan al derrumbe del modelo de mercado y la idea de "revolución" en ciernes. Dichas líneas se reconocen principalmente en: Oppliger y Guzmán (2012); Salazar (2011) y Mayol (2012 a y b). 
fundamentos del modelo económico ha dejado a la Concertación abierta a las críticas sobre que nada ha cambiado con la democracia" (2009: 16). Esta visión es reconocida por Flisfisch, Solari y Villar (2009), siendo los dos primeros personeros relevantes en los veinte años de gobierno de la Concertación:

(Destaca) La notable continuidad en la política económica (del gobierno de Aylwin ... (respecto de) la conducción económica autoritaria desde la segunda mitad de los años 80. Esa continuidad se explica mucho más como el resultado de una decisión política crucial ... Posteriormente, en los gobiernos de Frei y Lagos, la continuidad en los rasgos básicos de la conducción económica se explica atendiendo a otros factores. Uno es ciertamente la institucionalización de un establishment tecnocrático, favorecidas por entornos cupulares favorables a dichas concepciones, como fueron los que caracterizaron a esos gobiernos.

\section{El partido transversal, el cuoteo y la defensa del modelo}

El partido transversal y el cuoteo se constituyeron en las instituciones informales principales que contribuyeron en forma decisiva a neutralizar los obstáculos que el presidencialismo ponía a la operación de gobiernos de coalición, así como a la tensión entre las aspiraciones a la modificación del modelo económico y político heredado y su consolidación a lo largo de los gobiernos de la Concertación. Sin embargo, estos elementos entraron en crisis cuando generaron dificultades al sistema formal de partidos, cuando el cuoteo dejó de reflejar las correlaciones de fuerza internas de las colectividades extremando su carácter meramente formal y cuando el modelo económico, otrora "enclave central en el mantenimiento de la transición democrática" (Siavelis, 2009: 16), comienza a ser cuestionado por parte de la ciudadanía en aspectos tales como el rechazo a los abusos por parte del sector privado y al lucro en la educación.

Helmke y Levitski (2004:727) entienden por “instituciones informales” las reglas y los procedimientos, insistiendo en la necesidad de no confundir con "organizaciones informales", de manera de separar los actores políticos o jugadores de las reglas que ellos siguen. En ese sentido, cabe distinguir el concepto de "partido transversal" el que, siguiendo a Siavelis $(2009)^{5}$, es "el grupo informal integrado por políticos

Siavelis toma como referencia el concepto de Garretón (2003) de "enclaves autoritarios" para desarrollar el de enclaves de la transición. Avanza en identificar cinco, que considera fundamentales. Junto a los mencionados cuoteo e intocabilidad del modelo, menciona el control de la élite en la selección de candidatos, la dominación de los partidos en la política y la formulación de políticas elitista y extrainstitucional. 
clave" de las reglas a que se ajustaba el comportamiento de ese grupo, entre las cuales destacan el cuoteo y la defensa del modelo. Así, el partido transversal está constituido por una red de relaciones que incluye a militantes de los distintos partidos de la Concertación y a personeros independientes que compartían una historia común construida en la lucha contra la dictadura. En esta organización informal juegan un papel crucial personeros de izquierda que habían militado en la Democracia Cristiana (DC) y que mantuvieron y desarrollaron vínculos con miembros de dicho partido. A esta red se sumarán luego miembros de los principales centros de pensamiento que desarrollaron paradojalmente la crítica de la política económica y social de la dictadura ${ }^{6}$.

Sin embargo, también subyace al debate político una concepción más compleja del partido transversal que lo percibe como una estructura flexible que operaba al interior de los sucesivos gobiernos, pero con vínculos fuertes con la oposición política mediada de forma inédita por el importante rol intelectual y político de los centros de pensamiento vinculados a la derecha ${ }^{7}$ y los grupos dirigentes del gran empresariado. Augusto Varas (2012: 88 y ss) así lo señala:

El relevado "bloque en el poder" (se refiere a quienes concentraron el poder político, económico y social durante los 17 años de la dictadura) se transforma en poder fáctico, o lo que Gramsci llamó "cuasi partido" y Buci-Glucksmann conceptualizó como "partido oficioso", esto es la constelación interconectada, no institucionalizada y estable de las elites de poder en torno a una común visión de la sociedad organizada en torno a valores conservadores. Este eje estructurante le dio un fuerte carácter normativo a estas élites de poder... se organiza, según Antonio Cortés Terzi, como circuito extra institucional del poder. Este circuito - que no es una organización centralizada-observa tensiones internas y decisiones no siempre únicas, utiliza recursos no convencionales -privativos y de acceso privado-, interviene premeditadamente en lo público con funciones de organización $y$ dirección política sin disponer de organismos para ejercitar el poder en este espacio ni tener legitimidad democrática para ello ... Por otra parte, la gestión concertacionista a través de sus cuatro gobiernos generó sus propias elites en el poder, algunas de las cuales circularon entre el

6 Sobre el rol de los miembros de estos centros en el desarrollo político de los últimos años, ver Silva, 2010, y Joignant y Güell, 2011.

7 Lanzaro (2008) recuerda "el cultivo de una política de los consensos con el bloque de la derecha aun disponiendo de mayoría parlamentaria”. No hay que olvidar que la Concertación tuvo mayoría durante el primer año de gobierno de Michelle Bachelet, en 2006. 
mundo privado y estatal con cierta fluidez, sirviendo de puente entre el poder fáctico y el mundo estatal, especialmente en las instituciones reguladoras de la acción de las empresas privadas.

La colaboración trasciende con creces la institucionalidad reguladora. Más aún, es incluso más relevante cuando la colaboración se establece directamente entre organismos empresariales y las autoridades del gobierno en torno a iniciativas tendientes a producir efectos legislativos. Un ejemplo de este tipo de operación se estructura en el año 2001 a partir de la propuesta del presidente de la SOFOFA de concordar con el gobierno una Agenda Pro Crecimiento, generando amplios grupos de trabajo con los ministerios correspondientes para enfrentar los desafios económicos ${ }^{8}$. Del trabajo en común se desprenden diversas iniciativas, tanto legislativas como de carácter administrativo, que se presentan al Congreso y a los partidos de la Concertación para su aprobación (Siavelis, 2009:13). La colaboración exclusiva con el empresariado y la derecha va a adquirir mayor significación a partir de la crisis que vive el gobierno de Ricardo Lagos como efecto del llamado caso MOP-GATE. El acuerdo político alcanzado por el Ministro del Interior y el entonces senador y líder de la UDI, Pablo Longueira, para superar la crisis promoviendo iniciativas consensuadas en torno a la modernización del Estado inaugura modalidades y dispositivos de cooperación que terminarán imponiéndose a las estructuras partidarias de la Concertación. Elemento crucial es la visión compartida de la economía y de la política entre las autoridades gubernamentales y los centros académicos que constituían la base intelectual de la derecha (Fernández Ramil, 2010). Este proceso se desarrolló paralelamente a un debilitamiento de los partidos políticos de la Concertación, tanto como efecto del sistema político y los cambios en la base económica de la democracia (Huneeus, 2008) que afectaron su capacidad de incidencia efectiva en la formulación de las políticas públicas, como también por la creciente "presidencialización" del debate político como producto, entre otros factores, del acortamiento del mandato presidencial a cuatro años, sin reelección.

El partido transversal, bajo esta connotación amplia, alcanzaría su máximo desarrollo bajo la administración Bachelet gracias a la proliferación de comisiones asesoras presidenciales destinadas a "enfrentar los problemas de representación política, abriendo espacios alternativos en los que se incorporan nuevas voces y actores

Para una descripción de la propuesta y su alcance, consultar: http://www.sofofa.cl/mantenedor/ detalle. asp? $\mathrm{p}=1 \& \mathrm{~s}=2 \& \mathrm{n}=731$ 
sociales" (Aguilera, 2009: 11). Se trata de grupos de trabajo creados en torno a temas programáticos cruciales o para abordar temas generados por conflictos sociales agudos. Dos son sus aspectos más relevantes desde el punto de vista de la discusión en torno al partido transversal: por una parte, se buscaba un acuerdo "técnico" con la participación de especialistas identificados, tanto con el gobierno como con la oposición, para que los proyectos elaborados llegaran al Congreso con las líneas matrices consensuadas y, por otro, la importante participación de los centros de pensamiento vinculados al pensamiento más conservador de la Concertación y de la derecha, que conformaron el eje más poderoso de la toma de decisiones? ${ }^{9}$.

Este dispositivo de toma de decisiones respecto de las principales políticas públicas generó conflictos con los parlamentarios de gobierno. Aunque no es el factor fundamental, contribuye a explicar el éxodo de parlamentarios desde los partidos de la coalición, haciéndole perder al gobierno la mayoría por primera vez conquistada (Ominami, 2011:243). El fenómeno político del “díscolo” se multiplicó, expresando la crisis política de la coalición y alcanzando su clímax con la derrota de Eduardo Frei en la campaña presidencial del 2009-2010. A partir del 2006, con el gobierno de Michelle Bachelet, comienza a relativizarse la afirmación de Mainwaring y Shugart (2002: 263) según la cual “desde 1989, los partidos de la coalición electoral han sido miembros fieles de las coaliciones gobernantes de los presidentes".

\section{El partido transversal en la práctica: el nombramiento de los gabinetes}

Nombrar a sus ministros es una de las principales atribuciones presidenciales en Chile. Avendaño y Dávila (2012: 90) plantean que los gabinetes representan "el conjunto de agentes del ejecutivo designados políticamente en el nivel más alto del sistema de policy making. Asimismo, señalan que "constituyen un ámbito de gobierno en el que se definen buena parte de las políticas", permiten "vincular a los partidos con el gobierno" y "sus variaciones y criterios de designación permiten explicar las diferencias en policy making, entre otros aspectos que dan cuenta de su relevancia".

\footnotetext{
No es posible abordar este tema en detalle en el presente artículo. En el trabajo de Aguilera (2009:30) se muestra la numerosa participación de los centros indicados. Por otra parte, en el estudio de Rivera (2009:10) se muestra la posición dominante de la derecha en el Consejo para la reforma previsional. En la entrevista sostenida con el ex Ministro Francisco Vidal, este sostuvo taxativamente: "En el caso de Bachelet fueron importantes la comisiones presidenciales como gestoras de los proyectos de ley. Clave fue la composición desequilibrada que las caracterizó: estructuradas principalmente por Andrés Velasco fue abrumadora la mayoría fáctica de Libertad y Desarrollo, el Centro de Estudios Públicos, Expansiva y CIEPLAN. Se puede afirmar con certeza que fueron instrumento de las políticas conservadoras del entonces Ministro de Hacienda".
} 
El gobierno de Patricio Aylwin tuvo, como uno de sus objetivos, impedir un eventual retorno a un gobierno de facto. Clave era asegurar un crecimiento económico razonable, abordar los problemas de legitimidad asociados a los altos niveles de pobreza y consolidar el funcionamiento democrático. Diversos personeros manifestaron que la coalición delegaba en el presidente y su gabinete las decisiones en función de constituir un gobierno suprapartidario. Con ello se tomaba distancia respecto de los problemas que la incidencia de los partidos había tenido en el funcionamiento del gobierno de Salvador Allende. Apareció así la noción del partido transversal. No obstante, dicho partido fue primero una ficción. El presunto gobierno suprapartidario se estructuró sobre la base de un estricto cuoteo político que garantizó la participación proporcional en las responsabilidades del gobierno a los principales partidos. Gonzalo Martner sostiene:

El presidencialismo chileno es relativo; los quórums requeridos en el Congreso generan una situación de empate. En tal sentido, la influencia del Congreso es mayor de la que se estima normalmente. Lo más característico del sistema es que viabiliza el poder de veto de la minoría. Frente a la administración de Aylwin, si bien los partidos declararon el carácter suprapartidario del gobierno, lo cierto es que hubo una consulta estricta con los partidos en la configuración del gabinete. No hubo un ministro que no haya sido propuesto por los partidos. En suma, los ministros eran representantes del PS y nominados por los partidos en conversación con el Presidente de la República (Entrevista realizada el 6 de septiembre de 2012).

Existía, sin embargo, un sentido de cuerpo al interior del gobierno que generaba una dinámica relativamente independiente. En los últimos años de la lucha contra la dictadura y en la elaboración del programa de gobierno se generó una visión compartida y se crearon vínculos de confianza entre sus integrantes, lo que contribuyó a la consistencia y coherencia que imperó en las políticas impulsadas por el primer gobierno de la coalición. Las designaciones ministeriales realizadas correspondían a los personeros que habían jugado los roles más importantes en la estructuración de la Concertación y de la campaña, en la elaboración del programa y en el debate público. El análisis de la composición del gabinete deja en evidencia que se trata de personeros con un fuerte peso político propio y con incidencia en sus respectivos partidos.

Fue así como existió un equilibrio que reflejaba las votaciones parlamentarias de los distintos partidos de la coalición, una fluida relación con las organizaciones partidarias y, como luego se constataría, una clara coordinación con el Congreso. 
Más aún, característica de este primer gabinete fue la decisión de que, con sólo dos excepciones (los ministerios de Hacienda y Economía), los subsecretarios pertenecieran a partidos distintos de aquellos en que militaban los ministros. Esto deja en evidencia el compromiso con la coalición y permitía superar las dificultades que la existencia de múltiples partidos le generaba al sistema presidencial. El liderazgo político, la formación académica de los miembros del gabinete así como la solvencia técnica de los equipos ministeriales generó la percepción en los respectivos partidos y en su representación parlamentaria de que el Ejecutivo disponía de la visión estratégica y la capacidad de gestión que permitía resolver los complicados desafios de la transición y aseguraba la mantención de esos partidos en el poder en la medida que se sujetaran a las directivas gubernamentales.

Lo que bajo el gobierno del presidente Patricio Aylwin constituía una organización informal que le daba coherencia a la gestión de gobierno y a su colaboración con el Congreso, tuvo un posterior desarrollo que tendría importantes consecuencias políticas. En el año 1992, los democratacristianos del gobierno intentaron tomar directamente en sus manos el control de la estructura partidaria de la DC. Aprovechando la alta legitimidad del Presidente Aylwin y bajo el liderazgo intelectual de Edgardo Boeninger, se intentó levantar la candidatura de Alejandro Foxley, primero a la presidencia de la organización y, sobre esa base, a la Presidencia de la República. El asalto al partido desde el gobierno resultó un fracaso. La popularidad en la Democracia Cristiana del entonces senador Eduardo Frei y el poderoso grupo que lo acompañaba pudo controlar con rapidez el partido e iniciar, con ello, el camino hacia la Presidencia de la República.

El diseño inicial del gabinete del Presidente Frei $^{10}$ incorporó al equipo político de gobierno tres altos militantes de los principales partidos de la Concertación. Con ello se buscaba asegurar, tanto el compromiso partidario con el gobierno como la diversidad política de la coalición. En contraposición con el caso anterior, la relación fluida entre el nuevo gobierno y la coalición política que la sustentaba se estructuró a partir del rol e influencia que los nuevos ministros habían tenido en los principales partidos aliados a la DC. Fue así como se nombró a Germán Correa, presidente del Partido Socialista (PS), como Ministro del Interior y jefe de gabinete, y a Víctor Rebolledo, secretario general del Partido por la Democracia (PPD), como Ministro Secretario General de Gobierno. Completaban el equipo Genaro Arriagada y Carlos Figueroa que asumieron, respectivamente, el Ministerio Secretaría General de la

10 Sobre la administración de Eduardo Frei, ver Muñoz y Stefoni, 2003. 
Presidencia (SEGPRES) y el Ministerio de Relaciones Exteriores. Como es sabido, este diseño colapsó con rapidez. Dos elementos contribuyen a explicarlo. El primero es que si bien las afinidades personales no son indispensables, la colaboración previa es importante para el éxito de un gabinete. En contraposición con la situación anterior, los protagonistas no compartían historias comunes. En la primera reestructuración de su gabinete, Frei formalmente no altera el equilibrio político. El Ministerio del Interior es asumido por Carlos Figueroa (DC) (hasta ese entonces Ministro de Relaciones Exteriores), estrecho colaborador de su padre. La representación socialista se mantiene en una posición de primera importancia al asumir el cargo de Ministro de Relaciones Exteriores José Miguel Insulza. Lo mismo ocurre con la representación PPD, al asumir el Ministerio Secretaría General de Gobierno José Joaquín Brunner. Aun cuando persistía el cuoteo, la conformación del gabinete ministerial había cambiado sustancialmente. Los ministros salientes, pese a pertenecer al mismo partido, tenían una trayectoria con escasas vinculaciones con el partido transversal. En cambio, Insulza y Brunner habían sido democratacristianos y militado en el Movimiento de Acción Popular Unitaria (MAPU), originado de una escisión de la DC y, en consecuencia, tenía una gran red de relaciones con el partido dominante y el círculo presidencial.

Durante el gobierno de Patricio Aylwin, el partido transversal fue la expresión natural de la coordinación de los partidos en el gobierno y en su relación con el Congreso. Operó, en los términos de Helmke y Levitski (2004:728), como una organización informal complementaria del sistema formal de partidos. Como señalan los autores, bajo esta modalidad las instituciones u organizaciones informales "llenan los vacíos, ya sea al abordar contingencias no resueltas por las reglas formales o facilitando la búsqueda de fines individuales dentro del marco de las instituciones formales. Estas instituciones informales con frecuencia mejoran la eficiencia". Con el primer cambio de gabinete en el gobierno de Eduardo Frei, el cuoteo empieza a dejar de ser un mecanismo de equilibrio político entre partidos formales para constituirse en la cobertura de la distribución de cargos de una entidad que opera tras las bambalinas de los partidos formales. De esa forma, el partido transversal empieza a operar como lo que los autores indicados denominan instituciones informales acomodadizas (accommodating informal institutions), caracterizadas por operar en formas que alteran los efectos sustantivos de las reglas formales, pero sin violarlas directamente, esto es, las contradicen en su espíritu pero no en su letra (Helmke y Levitski, 2004: 729). Este cambio de naturaleza del partido transversal se mantendría bajo la administración de Ricardo Lagos. 
Por su trayectoria previa el presidente Ricardo Lagos (2000-2006) tenía un amplio conocimiento de las personas que formaban las redes de la Concertación y que podían formar parte de su gabinete. El protagonismo presidencial sería una constante en su administración ${ }^{11}$. Dos personeros parecen haber estado nominados muy anticipadamente: los futuros ministros de Hacienda y SEGPRES, Nicolás Eyzaguirre y Álvaro García, respectivamente. No obstante, no participaron en la nominación del resto del gabinete. Carlos Ominami (entrevista realizada el 5 de septiembre 2012) señala que preparó un listado de posibles miembros, aun cuando es probable que otros hayan realizado una tarea similar pues el Presidente solicitaba con frecuencia a diversos colaboradores la realización de las mismas tareas para disponer de alternativas en la toma de decisiones. Un nombramiento de particular importancia fue el del Ministro del Interior. José Miguel Insulza había jugado un papel clave en la revitalización del gobierno de Frei, afectado por el impacto de la crisis económica, por los problemas vinculados a la crisis eléctrica de 1998 - 1999, por la detención de Augusto Pinochet en Londres y por un debilitamiento relativo de la imagen global de la administración. Se trataba de un personero de larga y destacada vida política, con una vinculación permanente a la vida del PS y que había compartido con Ricardo Lagos en el gabinete del Presidente Frei. Pero además, Insulza personificaba la red de personeros provenientes de la DC, el PPD y el PS que, desde el gobierno, tomaban las principales decisiones relativamente al margen de las correlaciones de fuerza efectiva de los partidos.

Los personeros demócrata cristianos que se incorporan conforman dos grupos bastante diferentes. El primero, constituido por actores de larga trayectoria política y con importante influencia en el partido. Nos referimos a Soledad Alvear, ex ministra del SERNAM y de Justicia; Claudio Huepe, ex diputado e importante líder histórico de la tendencia de los "chascones" y Mariano Fernández, que asumen Relaciones Exteriores, Secretaría General de Gobierno y Defensa respectivamente, todos muy cercanos al Presidente y opositores al grupo que ejercería la dirección de la DC entre el 2002 - 2006. El segundo grupo estaba constituido por personeros conocidos y respetados en el partido, pero que carecían de influencia significativa, poseían un bajo perfil y tenían relaciones familiares directas con importantes dirigentes de la DC. Aunque con modalidades diversas, la nueva naturaleza que había adquirido el partido transversal en el gobierno de Frei se mantenía en la nueva administración.

11 Sobre las modalidades de operación de los presidentes ver Lahera (2008). 
En la entrevista sostenida con Gonzalo Martner éste destaca que, al ser el gobierno de Lagos la primera administración de izquierda desde Salvador Allende, el Presidente puso especial cuidado en que la nominación de los miembros democratacristianos de su gabinete garantizara una relación fluida con ese partido. Ello no era, sin embargo, una tarea fácil por el aumento de los conflictos en su interior. Baste recordar que, entre el año 1999 y el 2002, cinco personeros encabezaron el partido y que quien asumiría la conducción en el último año indicado lo hizo en una dura oposición con quienes hasta entonces lo dirigían. Pese a la incorporación al gabinete de personeros cercanos al nuevo presidente democratacristiano, en particular Jaime Ravinet en Vivienda, la coherencia entre el partido y los militantes en el gobierno operaba con dificultades. Esta situación se vio agravada por el retroceso electoral de la la DC en el año 2001 que acentuó la fragmentación y las lógicas particulares dentro del partido.

El gobierno de Michelle Bachelet, por su parte, se inicia con el triple compromiso de promover la rotación en el poder (llamado coloquialmente "la no repetición del plato"), asegurar la paridad de género y la promesa del "gobierno ciudadano". El primero de los compromisos apareció en el debate público como una respuesta a la crítica de la oposición de que, en caso de volver a ganar la Concertación, se produciría sólo un cambio de cargos pero el elenco gobernante sería el mismo. Con rapidez quedó en evidencia que la Presidenta sí cambió significativamente el personal político respecto de los gobiernos precedentes. Ello debilitó los lazos entre los partidos políticos de la coalición y la autoridad presidencial. La participación del PS se circunscribió principalmente a una de sus fracciones, la denominada "Nueva Izquierda", liderada por Camilo Escalona y a la que pertenecía la primera mandataria. Expresión de ello fueron los nombramientos de María Soledad Barría, que ocupó el Ministerio de Salud y Osvaldo Andrade, el de Trabajo y Previsión Social; Paulina Veloso en la SEGPRES y Clarisa Hardy en el Ministerio de Planificación. Esta última, en el marco de la Fundación Chile 21, había coordinado la preparación de buena parte de las bases de la política social de la nueva administración.

La participación del Partido Por la Democracia se limitó principalmente a la nominación de ministros vinculados al think tank Expansiva, liderado por el independiente Andrés Velasco: Eduardo Bitrán, en Obras Públicas y Vivian Blanlot, en Defensa. Completaba el grupo Karen Poniachick, en Minería y Energía. Al contrario del gobierno anterior y, en cierto sentido, a contrapelo de la aspiración a renovar los equipos, una parte del elenco de la DC estaba conformado por experimentados políticos liderados por Andrés Zaldivar, en Interior y Alejandro Foxley, en Relaciones Exteriores. Sin embargo, también se incorporaron figuras 
nuevas como Álvaro Rojas, rector de la Universidad de Talca en Agricultura; Martín Zilic, en Educación y Patricia Poblete, en Vivienda. Común a todos ellos fue su baja influencia en las principales decisiones del gobierno. En este contexto, el partido transversal experimentaba un cambio de personal y asumía un nuevo carácter. En relación con lo primero, se estructura un eje constituido por lo que hasta ese entonces había sido el sector más izquierdista del PS, excluido de las responsabilidades gubernamentales en los tres gobiernos anteriores, y el grupo Expansiva, que representa la autonomización política del componente liberal de la Concertación que, hasta ese entonces, había operado en funciones secundarias y sobre la base de su influencia técnica. Esto ocurría en momentos en que la ciudadanía empezaba a poner en cuestión el modelo económico y los ejes fundamentales de la propuesta concertacionista, todo lo cual se manifestaría en diferentes movilizaciones sociales (estudiantiles, subcontratistas, usuarios del sistema de transporte de personas de Santiago, entre otras), el surgimiento del fenómeno de los díscolos en el Congreso y la salida de personas y grupos de los partidos del conglomerado de gobierno.

De esta manera, continúa operando en el gobierno de coalición un partido transversal pero con una nueva composición, en tensión con buena parte de la militancia concertacionista, lo que plantea la hipótesis sobre la naturaleza acomodaticia de esta institución informal que empieza a presentar rasgos propios de las instituciones informales competitivas de Helmke y Levitsky (2004). Los continuos tropiezos que experimenta el gobierno, la alta conflictividad social y las pugnas internas llevan a la Presidenta de la República a modificar su gabinete al año de asumida, reinstalando personeros más afines con la red transversal de las administraciones anteriores. Si bien se logra mejorar la gestión gubernamental ${ }^{12}$, la conflictividad al interior de la coalición termina en la derrota en la elección presidencial del 2010 por cuanto su fuerza electoral se divide entre los candidatos Frei y Enriquez - Ominami.

\section{La Presidencia de Sebastián Piñera: Del gabinete personal al de coalición y la aparición de un nuevo partido transversal}

Al analizar el gobierno de Sebastián Piñera (2010-2014), los analistas pusieron énfasis en el sello que le imprimiría la personalidad del Presidente, abonado por características sui generis como empresario y por su lema central de campaña"la nueva forma de

12 Para un análisis del giro indicado ver Funk (2009). 
gobernar"13. Su primer gabinete reunió a sus colaboradores más cercanos. Rodrigo Hinzpeter y Felipe Larraín, en las posiciones claves de Interior y de Hacienda respectivamente; a conocidos suyos por sus negocios previos (como Jaime Mañalich, en Salud) y a académicos que había conocido en la labor programática de los grupos Tantauco, aunque carentes de experiencia política significativa (De Solminhiac, en el Ministerio de Obras Publicas; Morandé en el ministerio de Transportes y Telecomunicaciones y Rainieri, en el de Energía). La UDI contribuyó con el excandidato presidencial Joaquín Lavín, que venía de sufrir su tercera derrota política en la elección senatorial de la Quinta Región costa. Se incorporaron también Ena Von Baer, que también había perdido en una elección senatorial previo paso por Libertad y Desarrollo, cantera de la que también salió Felipe Kast, a cargo de Mideplan. En una variante de gobierno de cooptación ${ }^{14}$, incorporó a Jaime Ravinet, de la DC, a la cabeza de Defensa, animado por la idea de promover un gobierno con sentido de "unidad nacional". Como puede observarse de la evolución del nombramiento de gabinetes desde 1990, el carácter estrictamente partidista se fue diluyendo en las dos últimas administraciones así como lo que habría sido una coalición de estilo parlamentario que, de acuerdo a Garrido (2003, 58), habría permitido el funcionamiento del pacto con una mayor estabilidad ministerial que en otro tipo de gabinetes de coalición.

Al describir al gobierno, el entonces senador Longueira, afirmó que se trataba de un gobierno de Sebastián Piñera y no de la Coalición por el Cambio. Más aún, se trataba de un gobierno extremadamente personalista. En una entrevista realizada por los autores, éste señaló:

Yo creo que este es un gobierno que funciona... como eje principal... con la figura del presidente. Todo pasa por el presidente ... me atrevería a decir que creo que en todas las carteras, tiene una relación bilateral con cada ministerio donde él decide todo, hasta el mínimo detalle y eso se ha impregnado de tal forma que todos sienten que todo tiene que ser autorizado. O sea, se generó una estructura completamente vertical, $y$ en eso creo que no hay distingo entre carteras con ministros más políticos y con menos políticos. En cuanto a la toma

13 Héctor Soto es uno de los analistas que más se ha referido a la personalidad del Presidente Piñera: http://diario.latercera.com/2012/09/01/01/contenido/reportajes/25-117356-9-apuntes-paraentender-el-caracter-de-pinera.shtm (Accesado el 8/5/2013)

14 De acuerdo a la idea sugerida por Garrido (2003: 52) según la cual este tipo de gobiernos suponen la extensión de carteras ministeriales a ciertos miembros de dos o más partidos, sin alcanzar un acuerdo de colaboración en el gobierno con los dirigentes de estos partidos. 
de decisiones estoy hablando. Creo que hay una cosa muy estructurada".

(Entrevista realizada el 05 de septiembre de 2011).

El presidente Piñera nombró a cargo del equipo asesor directo (denominado desde el gobierno de Lagos, Segundo Piso) a María Luisa Brahm, con la que mantenía una relación de trabajo por largos años, y quien no solamente había sido directora ejecutiva del Instituto Libertad, sino que había integrado el consejo de la Alta Dirección Pública (ADP) en gobiernos concertacionistas. Dicha condición le entregaba un conocimiento particularmente profundo del aparato del Estado. Adicionalmente, se crea en el año 2010 la llamada Unidad de Cumplimiento de Gestión, dependiente del Ministerio Secretaría General de la Presidencia, inspirada en la Delivery Unit británica, como una forma de hacer seguimiento a las promesas presidenciales. Todo ello auguraba un funcionamiento, al menos, desde el punto de vista orgánico, más promisorio de lo que los eventos políticos vinieron posteriormente a demostrar.

La imposición por parte del Presidente de la República de un gabinete constituido al margen de las opiniones de las directivas y de los personeros más influyentes, tanto de la Unión Demócrata Independiente (UDI) como de Renovación Nacional (RN) generó, desde el principio, diversas críticas. Particular molestia demostraron Andrés Allamand y Pablo Longueira, principales figuras de RN y la UDI respectivamente, quienes resentían no haber sido incorporados. Posteriormente, la falta de experiencia política de sus integrantes se demostró en sucesos tales como el largo tiempo que se requirió para resolver la crisis generada en torno a la Intendenta de la región del Bío Bío; el rol del Ministro de Energía en el desencadenamiento de las movilización en Punta Arenas; la crisis en torno a los pagos realizados por el Ministerio de Vivienda a una empresa constructora, llamado “caso Kodama” y el estallido social en Aysén, entre otras.

El primer mandatario buscó mantener una relación de trabajo permanente con sus ministros. Para ello, estructuró su entorno sobre la base de tres equipos de asesores. Un equipo asesor inmediato, la Asesoría Presidencial, dirigido por María Luisa Brahm; un equipo asesor coordinador tanto en lo jurídico como en la gestión, radicado en la Secretaría General de la Presidencia (SEGPRES), y un equipo financiero presupuestario constituido por la Dirección de Presupuesto del Ministerio de Hacienda (DIPRES).

En una posición altamente influyente se encuentra la Asesoría Presidencial. Respecto a su origen y accionar José Miguel Izquierdo, uno de sus miembros, señalaba: 
Lagos dio un consejo al presidente Piñera, en el cual le dijo 'tenga un Segundo Piso poderoso, porque los ministros tienen su agenda propia', y eso se ha ido reforzando en el transcurso de este año y medio. Pero hoy día, lo del seguimiento $y$ de la coordinación interministerial es totalmente participativa, $y$ nosotros nos nutrimos de su información $y$, cuando vemos que están flaqueando en algún sector, nosotros los apretamos ahí. Los apretamos a ellos. Los apretamos a los ministerios. Y otra razón por la cual no está ocurriendo esto de que no se observan en los detalles es el sistema de gobierno del presidente que son las bilaterales. Pero es... en el fondo, este atributo 'Piñera gerente' se despliega en las bilaterales, $y$ en estas bilaterales nosotros cumplimos una función muy importante. Que es la preparación de los técnicos y de los ministros para reunirse con el presidente. Para lograr el estándar que el presidente exige. $Y$ luego... ¿con el staff de los ministros? Sí, nosotros estamos encima. Por ejemplo, yo ahora estoy preparando una bilateral de defensa. Entonces, ahora a las 15:30 tengo reunión con el ministro, porque el presidente está un poco tostado con un par de temas. Entonces, a cantarle al ministro. El segundo piso, este segundo piso, tiene bastante poder. $Y$ ha generado, está generando, una reacción en ese sentido. Porque los ministros se sienten... [Entrevistador: ¿Auscultados...?] Sí, tienen a alguien que les está tirando las orejas, que es el presidente. El hecho de que uno llame en nombre del presidente se siente... violento, como ministro" (Entrevista realizada el 5 de abril de 2011).

El objetivo asignado por el Presidente a los grupos de asesores era asegurar la coherencia presupuestaria y programática. La modalidad de trabajo descrita buscaba permitir una supervisión directa de las tareas desarrolladas por cada ministerio, su consistencia con los objetivos del gobierno y el avance simultáneo y coordinado de conjunto del plan de acción. La conducción presidencial y su coordinación con los diferentes ministerios estaban estructuradas sobre la base de las llamadas reuniones bilaterales que reunían al presidente, acompañado por los tres equipos de asesores indicados, $\mathrm{y}$ al ministro correspondiente.

Al analizar la forma de operar la toma de decisiones llama la atención que, según informaciones oficiales, las reuniones bilaterales alcanzan un promedio de cuatro reuniones por semana. Su frecuencia plantea una hipótesis acerca del estilo de gestión del presidente, que favorecería un trabajo un trabajo estructurado sobre la base de un relacionamiento más personal y directo entre el primer mandatario y sus ministros, en perjuicio de la lógica más colectiva propia de los comités interministeriales (como era el caso en las administraciones anteriores). En 
diversos artículos de prensa, la información sobre esta modalidad de operación del Presidente se presentó como propia de uno muy involucrado con la gestión cotidiana, que condicionaba a sus ministros a una actividad intensa y a continuos "exámenes", aprovechando su alto conocimiento de los temas. Genaro Arriagada ${ }^{15}$ sostuvo que la presidencia de Piñera estaba caracterizada por el micromanagement, entendiendo por tal una de tipo gerencial donde el jefe de gobierno, estableciendo las funciones administrativas como las principales, procura tener bajo completo control las funciones de la administración. Frente a esta visión, Arriagada propuso el concepto alternativo y necesario de una presidencia que debiera poner el acento en las tareas políticas.

Si bien cabe coincidir con esta perspectiva, dichas características son un síntoma de una visión más global que imperaba en el gobierno y según la cual su ejercicio estaría disociado en dos partes, práctica y conceptualmente separadas: la gestión de las políticas, que aparece como el problema sustantivo a resolver, y la gestión de la política percibida más como un problema que como el medio de organización de la vida en sociedad. La separación entre el concepto de politics como separado de policies fue el eje de la gestión en la primera fase de la administración, pudiendo ser descrita de la siguiente forma: mientras los políticos se dedican - y pierden el tiempo- al corto plazo y a la pelea chica, otros (nosotros) estamos construyendo las políticas de Estado. Aplicado a la gestión gubernamental, esto se traduce en que el Presidente dedica parte sustancial de su tiempo en la "gestión de la gestión" con múltiples reuniones bilaterales, "delegando" en figuras, cuyo liderazgo es poco consolidado, la "gestión política". El predominio del presidente al interior del Ejecutivo contrastaba con la ausencia de liderazgo del Ejecutivo en la agenda política y en la sociedad (Varas, 2012b).

La incorporación al gabinete, primero de Andrés Allamand y Evelyn Matthei, y luego de Longueira y Chadwick, a mediados del año 2011, puede ser interpretada como la resolución del conflicto entre técnicos y políticos. El eje analítico del presente artículo retoma esta idea, pero propone un matiz crucial: la negociación que llevó a Pablo Longueira a ingresar al gabinete no sólo suponía reconocer la importancia de los políticos sino que, además, representaba un cambio fundamental en la estructuración del gobierno al sustituir un tipo de presidencia gerencial y relativamente autónoma de su base política partidista por una conducción política eficaz basada en el compromiso

15 Así lo señala en "Creo que Piñera ha dañado fuertemente la imagen presidencial", en http://m. df.cl/genaro-arriagada-creo-que-pinera-ha-danado-fuertemente-la-imagen-presidencial/prontus_ df/2011-07-14/214339.html (Accesado el 12 de mayo de 2013). 
de Longueira de ordenar a la UDI en torno al gobierno. Este proceso comprometía el apoyo de la UDI y, con ello, se buscaba resolver las tensiones entre el régimen presidencial y la presencia de una coalición de partidos.

Junto con el debate en torno al rol de los partidos en la coalición había surgido la discusión respecto de si el gobierno estaba orientando sus políticas sobre la base de las convicciones del sector o si, por el contrario, se habían adoptado las ideas de la Concertación, como sostenían una serie de personalidades que habían sido ministros de la dictadura militar y personeros del think tank de derecha "Libertad y Desarrollo”. Esta situación sugiere una hipótesis adicional.

Mientras el protagonista de la rebelión fue la UDI, el sector más ortodoxo de la derecha mantenía esperanzas de que, teniendo ese partido éxito con sus presiones, podría introducirse un cambio en las prioridades gubernamentales desde un énfasis social a la priorización de lo económico, considerado como preocupaciones propias de ese sector político. Con la incorporación de Longueira y Chadwick ello no ocurrió (como tampoco había ocurrido al incorporarse Evelyn Matthei, que comenzó mostrando celo en el cumplimiento de la legislación laboral, resistido por sectores del mundo empresarial). Longueira apoyó decididamente las reformas sociales en marcha, esgrimiendo un fuerte discurso contra el abuso empresarial y en favor de una activa política de protección al consumidor. Ello proveyó al gobierno de un relato que se había desdibujado y que, con un presidente poco diligente en la resolución de sus conflictos de interés, no resultaba del todo creíble. Este énfasis le dio más fuerza y un mayor contenido a los planteamientos de Hinzpeter, formulados en el segundo semestre del 2010, sobre la oportunidad y la necesidad de construir una nueva derecha. En el fondo, al liberalismo político propugnado por los partidarios de la nueva derecha, se sumaba algo que se podría denominar intervencionismo del "libre" funcionamiento del mercado. Este discurso, y las políticas asociadas a ello, generaron preocupación en un sector de la UDI (encabezado por el senador Jovino Novoa); en ex-ministros de la dictadura de Pinochet (específicamente, Rolf Lüders y Hernán Büchi) ${ }^{16}$; y en algunos dirigentes empresariales, entre otros.

Aunque todavía resulta temprano para presentar análisis definitivos, sí resulta plausible asumir que la transición desde un gobierno personalista característico de la primera fase de la administración de Piñera hacia un gobierno de coalición se hizo posible por la constitución de una organización informal sujeta a normas propias que

\footnotetext{
16 Para una revisión detallada de los conflictos en la derecha en torno a las orientaciones del gobierno, verVaras (2012 b).
} 
empezó a definir los destinos del gobierno, de sus políticas y de los partidos de la coalición. Fortaleciendo la dimensión del diálogo y la operación conjunta la derecha modifica, parcialmente, la trayectoria de conflictos entre la UDI y RN sobre la base de una red constituida por personeros de ambos partidos, liderados por el propio Presidente de la República y algunos de sus principales ministros, que enfrentan a los sectores más conservadores de ambos partidos estableciendo, al mismo tiempo, mayores distancias con el mundo empresarial. Este proceso era necesario para avanzar hacia una modernización de ese sector político, que seguía desentonando con los nuevos escenarios, no sólo del país, sino a nivel internacional. Otros indicadores de este proceso son, por un lado, la persistencia del mayor celo con que el gobierno y la derecha han venido tratando los abusos empresariales, que tuvo su punto culminante en el desplome de la candidatura presidencial de Laurence Golborne, abanderado de la UDI. Por otro, la forma como tanto Andrés Allamand como Pablo Longueira apostaron a mantener la primaria presidencial en la coalición de derecha, pese al grado de beligerancia que existía hasta ese momento entre sus dos tiendas. Retomando los conceptos de Helmke y Levitski (2004), una institución informal "acomodante" alteraba los efectos de las instituciones formales, pero sin violarlas.

\section{El funcionamiento de los gabinetes y el creciente poder de los Ministros de Hacienda}

Para entender los mecanismos informales en el presidencialismo chileno, no basta poner el acento en la conformación inicial de los gabinetes y sus características matrices, sino que es importante también observar las lógicas de su funcionamiento en relación al papel asignado, en el caso chileno, a una cartera particular, la de Hacienda.

Entre los enclaves de la transición, Siavelis (2009) alude, como ya se señaló, a la intocabilidad del modelo económico, llamando la atención a que los actores de todo el espectro político concordaban en que parte del acuerdo no escrito aseguraba que, en la transición, el modelo económico debería permanecer sin modificaciones. Ese compromiso, según dicho autor, habría tranquilizado a las elites económicas "cuya reacción a un posible cambio en la política podría haber sido extraordinariamente desestabilizadora". Este compromiso encuentra en primer lugar su expresión formal en las reformas constitucionales que sancionan los enclaves autoritarios (Garretón, 2003) y que refrendan un escenario hostil a la transformación del modelo. En este contexto, el Ministro de Hacienda va a asumir un rol clave en garantizar que la política pública no lo va a poner en cuestión y que, por el contrario, buscará su perfeccionamiento. 
En el caso del gobierno de Aylwin, la coordinación gubernamental se concentró en el Ministerio Secretaría General de la Presidencia (SEGPRES), tanto en lo relativo a la coherencia interna del Ejecutivo como en sus relaciones con los partidos políticos y el Congreso. El impulso de la agenda se basó en una estrecha relación entre este ministerio y el de Hacienda, dada "la gravitación principal que desde el primer momento se reconoció en dicho ministerio responsable de la política económica" (Boeninger, 2007:53). Debido a la naturaleza pluripartidista que tenía la base política del gobierno, se incorporó a "esta férrea unión” el Ministro de la Secretaría General de Gobierno, de militancia socialista, dándole a la alianza SEGPRES Hacienda un carácter transversal. Este modelo de conducción se complementó con los comités interministeriales y las comisiones bipartitas Ejecutivo - Parlamento. A ellos se sumaban asesores que tenían como objetivo el establecimiento de vínculos sistemáticos entre políticos y tecnócratas (Boeninger, 2007: 53).

Boeninger estaba convencido de que el éxito de la transición dependía de la capacidad de asegurar una adecuada conducción económica que, además, generara confianza en el mundo empresarial, cuya colaboración era considerada clave para la consecución exitosa de la transición. En tal sentido, señalaba que era "esencial otorgar y mantener un fuerte respaldo presidencial y de los ministros políticos claves al ministerio conductor de la política económica, como condición política de continuidad, predictibilidad y estabilidad de la economía" (Boeninger, 2007: 55). El acceso de Eduardo Frei al gobierno estuvo determinado por el éxito económico alcanzado bajo la administración precedente. Su amplio triunfo reflejó la satisfacción generalizada por haber sido posible reunir con éxito la democracia con la prosperidad económica. En este contexto, Manuel Antonio Garretón sostiene lo siguiente:

Lo que estaba en juego al iniciarse el gobierno de Frei era el modo como se enfrentaría en conjunto la continuidad de la democratización política, es decir las tareas pendientes de la transición, la transformación social que apuntara a la superación de la pobreza y, sobre todo, de las desigualdades sociales, lo que exigía, a su vez, la reformulación del modelo de desarrollo y reinserción mundial $\gamma$, finalmente, la generación colectiva de un modelo de modernidad $\gamma$ convivencia que asegurara tanto la diversidad socio-cultural como la unidad $y$ cohesión nacional. El error del gobierno fue plantearse prioridades entre estos aspectos o dar algunas de estas tareas como finalizadas, lo que llevó de hecho a definir el crecimiento económico como la única tarea decisiva y a privilegiar, entonces, al actor empresarial y la conducción de Hacienda. (p. 52). 
La declaración del fin de la transición a la democracia y la proposición de hacer de la modernización el eje de la gestión gubernamental abrió paso a un predominio renovado y de nuevo tipo del Ministro de Hacienda que permitió fortalecer adicionalmente el ministerio como tal y, en particular, la Dirección de Presupuestos (DIPRES), en el Ejecutivo. No cabe duda que el rol de estas entidades en relación con el presupuesto y las obligaciones de preparar un informe financiero sobre todas las iniciativas legales le aseguraban una posición de alta influencia, más allá de sus funciones específicas. La introducción de mecanismos de control de desempeño y de evaluación de programas sociales le permite construir un fuerte aparato de seguimiento de toda la administración y un amplio dispositivo de intervención en áreas de la gestión pública sobre las cuales no tenía incidencia anteriormente. Esto hizo posible generar una base de influencia en las políticas públicas para el Ministerio de Hacienda y para la DIPRES que tiene la potencialidad de operar casi independientemente de la fuerza que, en las distintas coyunturas, pueda tener el Ministro de Hacienda de turno ${ }^{17}$. Se terminó produciendo lo que Agostini (2007) advierte como un problema de diseño institucional: dicho ministerio actúa como "gerente de operaciones" más que como "gerente de finanzas".

El gobierno del Presidente Ricardo Lagos combinó un Presidente altamente conocedor de todos los temas; un Ministro del Interior como jefe político, el Ministro de Hacienda, crecientemente involucrado en los temas políticos y sectoriales y el llamado "Segundo Piso", compuesto por asesores presidenciales directos que tomaban diversas iniciativas, apoyaban directamente al Presidente y establecían una alianza con el Ministro de Hacienda, realizando desde esta perspectiva una labor similar a la SEGPRES en el primer período" (Boeninger, 2007: 53 - 54).

En el contexto de estos entramados en competencia, se inició una lucha por influir en el primer mandatario. Álvaro García, de la SEGPRES y Nicolás Eyzaguirre, de Hacienda, tenían una larga trayectoria de amistad y cercanía con el Presidente de la República. Dos temas parecen haber constituido los nudos claves en el conflicto que terminaría con la salida del primero: la orientación y la conducción de la reforma del Estado, a la cual el Presidente le dio una gran importancia en la primera fase de su gobierno, y lo relativo a la radicación de la evaluación de programas y políticas públicas. Probablemente, la constitución de una estrecha alianza entre el Ministro del Interior, el de Hacienda y el "Segundo Piso" contribuyó a que el conflicto se resolviera a favor del Ministerio de Hacienda. Esto marcaría la

17 Para profundizar este tema, se sugiere consultar Aninat y Rivera, 2009: 107 y ss. 
conducción gubernamental. Lo político quedaría en manos del Ministro del Interior y la conducción económica y conducción operativa de la gestión gubernamental pasarían a manos del ministerio de Hacienda. Es probable, sin embargo, que la salida de Álvaro García representara también el fin de una modalidad de relaciones entre el gobierno y los principales actores sociales. En efecto, desde el gobierno de Frei, Álvaro García había venido promoviendo un diálogo tripartito que se expresó en encuentros anuales denominados Foros de Desarrollo Productivo, en los que confluían y debatían un gran número de dirigentes empresariales y de los trabajadores con las principales autoridades del gobierno ${ }^{18}$. En ese contexto, se aprobó, por ejemplo, un programa de tres años de reajuste de salario mínimo que implicaría una mejora real sustantiva. Al asumir como Ministro Secretario General de la Presidencia en la administración de Ricardo Lagos, Álvaro García buscó retomar el diálogo tripartito, ahora desde el ministerio indicado, para proyectar el diálogo social hacia los desafios más generales del país. No obstante, esta política no avanzó. Al abandonar el gabinete, el diálogo gobierno - sociedad civil adquiriría una nueva fisonomía, esta vez bajo los auspicios de Nicolás Eyzaguirre, Ministro de Hacienda. En efecto, a principios de marzo (apenas dos meses después de la salida de García), dicho Ministro dio a conocer la Agenda Pro Crecimiento preparada por los "académicos y técnicos del sector privado, así como los expertos del sector público que durante 60 días trabajaron con el objetivo de promover el crecimiento del país" ${ }^{19}$. Asistieron al evento el Presidente de la República y los principales dirigentes empresariales pero nadie del movimiento sindical. El Ministro de Hacienda concordaba así, con los representantes del empresariado, las líneas básicas de la política económica ${ }^{20}$. La salida simultánea de Carlos Cruz del Ministerio de Obras Públicas y su reemplazo por el entonces Director del Servicio de Impuestos Internos, Javier Etcheberry, representaría la llamada al orden del único ministerio sectorial que se escapaba del férreo control ejercido por el Ministerio de Hacienda.

18 Sobre este tema ver french-Davis y Muñoz, 2003, pp. 278 y ss.

19 Para profundizar sobre este tema se recomienda revisar: http://www.emol.com/noticias/ economia/2002/03/15/80945/gobierno-anuncio-primeras-propuestas-de-la-agenda-procrecimiento.html (Accesado 26/5/2013)

20 Desde una mirada más amplia, se puede argumentar que la Agenda Pro Crecimiento surge como efecto de la creciente influencia de Juan Claro en el mundo empresarial (primero en la SOFOFA y luego en la Confederación de la Producción y el Comercio) y como respuesta a la crisis económica y la tensión de Lagos con los empresarios al comienzo del gobierno: "déjennos trabajar tranquilos" de Ariztía (http://www.emol.com/noticias/economia/2001/11/13/71131/empresarios-emplazana-ministro-solari-a-decidir-entre-gobierno-y-la-cut.html). No obstante, la resolución del conflicto entre Eyzaguirre y García coincide con el abandono de la prioridad asignada a la colaboración tripartita en beneficio de los acuerdos gobierno - cúpulas empresariales. 
El posicionamiento de Andrés Velasco como primera figura del gabinete durante el gobierno de Michelle Bachelet se hizo posible, entre otros factores, por el fortalecimiento que había experimentado el Ministerio de Hacienda respecto de Nicolás Eyzaguirre como articulación entre el gran empresariado y el gobierno y por el apoyo irrestricto que le entregó la Nueva Izquierda, grupo dominante en el PS y en la coalición, para cumplir esta tarea. Esto explica el predominio de una política que tuvo como eje el perfeccionamiento del modelo lo que generó, como se señaló más arriba, una grave crisis en la coalición de gobierno. En el gobierno de Piñera pierde relevancia el rol del Ministro de Hacienda puesto que es el propio Presidente de la República el que garantiza la continuidad del modelo.

\section{Conclusiones}

El carácter exploratorio del trabajo permite adelantar algunas hipótesis. Por medio de un análisis comparativo de los procesos de designación de los gabinetes ministeriales y de su toma de decisiones, centrado en los cinco gobiernos desde 1990 a la fecha, en Chile quedan en evidencia "mecanismos institucionales informales", entendidos como dispositivos que permiten adaptar los rígidos mecanismos formales a las realidades políticas, aun cuando en ocasiones sirven para encubrir el efecto de otros mecanismos. Bajo Aylwin, el partido transversal fue la expresión natural de la coordinación de los partidos en el gobierno y en su relación con el Congreso. Operó en los términos de Helmke y Levitski (2004), como una organización informal complementaria del sistema formal de partidos. Bajo Frei y Lagos, el partido transversal empieza a operar en la modalidad de instituciones informales acomodantes (accommodating informal institutions), caracterizadas por operar en formas que alteran los efectos sustantivos de las reglas formales, pero sin violarlas directamente, esto es, las contradicen en su espíritu pero no en su letra. Bajo Bachelet continúa operando en el gobierno de coalición un partido transversal, pero con una nueva composición en tensión con buena parte del establishment concertacionista, lo que plantea la hipótesis sobre la naturaleza acomodaticia de esta institución informal que empieza a presentar rasgos propios de las instituciones informales competitivas de Helmke y Levitsky (2004). La transición desde un gobierno personalista, característico de la primera fase de la administración de Piñera hacia un gobierno de protocoalición, se hizo posible por la constitución de una organización informal sujeta a normas propias que empezó a definir los destinos del gobierno y sus políticas y por encima de los partidos de la coalición. Finalmente, el artículo indaga en el rol clave que asume el Ministerio de Hacienda y quien lo detenta en la preservación del modelo, institución informal que orientó y orienta los gobiernos desde 1990. La indagación, 
a partir del caso chileno, arroja antecedentes que debieran considerarse en la revisión crítica de la importancia asignada a los aspectos formales del presidencialismo.

\section{Referencias bibliográficas}

Agostini, Claudio (2007, diciembre). El rol del Ministerio de Hacienda: ¿Gerencia de finanzas o de operaciones? Recuperado 8 de mayo de 2013, de: http://www.blogeconomia.uahurtado. $\mathrm{cl} / ? \mathrm{p}=22$

Aguilera, Carolina (2009). Un análisis de las comisiones asesoras presidenciales del gobierno de Michelle Bachelet. Documento de trabajo Programa de Gobernabilidad. Santiago: FLACSO.

Aninat, Cristóbal y Eugenio Rivera (2009). "Coordinación estratégica en el Estado de Chile", en Tomicic,Visnja y García, Cristián (coordinadores y editores). Un mejor Estado para Chile. Propuestas de modernización y reforma. Santiago: Consorcio para la reforma del Estado.

Avendaño, Octavio y Dávila, Mireya (2012). "Rotación Ministerial y estabilidad coalicional en Chile; 1990 - 2010”. Política, Revista de Ciencia Política. Vol. 50, N² 2, pp. 87-108.

Barómetro de Política y Equidad (2012). A mitad de camino. Punto de no retorno. Santiago: Fundación Equitas/ Fundación Friedrich Ebert.

Boeninger, Edgardo (2007). Políticas públicas en democracia. Institucionalidad y experiencia chilena 1990 - 2006. Santiago: Uqbar Editores.

Cavallo, Ascanio (2012, agosto). "El núcleo estratégico del gobierno”. Recuperado el 13 de agosto de 2012, de: http://blog.latercera.com/blog/acavallo/entry/el_n\%C3\%BAcleo_ estrat\%C3\%A9gico_de_gobierno

Díaz-Tendero, Eolo (2006). "Iluminismo democrático "versus" ciudadanía sectorial”. En Pérez Herrero, Pedro (editores). La "izquierda” en América Latina. Madrid: Editorial Pablo Iglesias.

Fernández Ramil, María de los Ángeles (2010, febrero). Think tank y Concertación: No basta con llorar. Recuperado el 27 de mayo de 2013, de: http://www.elmostrador.cl/ opinion/2010/02/22/el-hacer-opositor-y-la-oportunidad-para-el-saber-experto/

Fernández Ramil, María de los Ángeles y Eugenio Rivera Urrutia (2012). La trastienda del gobierno. El eslabón perdido en la modernización del Estado chileno. Santiago: Editorial Catalonia.

Ffrench-Davis, Ricardo y Muñoz, Oscar (2003). "Las políticas económicas y sus efectos", en Óscar Muñoz y Carolina Stefoni (coords.). El período del Presidente Frei Ruiz-Tagle. Santiago: Editorial Universitaria.

Flisfisch,Ángel; Solari, Ricardo y y AndrésVillar (2009). "Las siete vidas de la Concertación”, en Carlos Bascuñán, Germán Correa,Jorge Maldonado yVicente Sánchez (Editores). Más acá de los sueños, más allá de lo posible. La Concertación en Chile. Vol. 1, Santiago: LOM Ediciones. 
Funk, Robert (2009). “Chile: segundo tiempo”. Revista de Ciencia Política. Vol. 29, N², pp. 301-326.

Garretón, Manuel Antonio (2003 A). Incomplete Democracy. Chapel Hill: University of North Carolina Press.

Garretón, Manuel Antonio (2003 B). "La (in)conducción política del Segundo Gobierno Democrático”, en Óscar Muñoz y Carolina Stefoni. El período del Presidente Frei Ruiz Tagle. Santiago: Flacso-Editorial Universitaria.

Garrido, Antonio (2003). “Gobiernos y estrategias de coalición en democracias presidenciales: el caso de América Latina”. Política y Sociedad.Vol. 40, º 2, pp. 41-62.

Helmke, Gretchen y Levitsky, Steven (2004). "Informal Institutions and Comparative Politics: A Research Agenda”. Perspectives on Politics.Vol. 2, N 4, pp. 725-740.

Huneeus, Carlos (2008). “¿Hacia una democracia corporativista?” Revista Mensaje. Mayo, pp. $8-13$.

Joignant, Alfredo y Güell, Pedro (Editores) (2011). Notables, tecnócratas y mandarines. Elementos de sociología de las élites en Chile (1990 - 2010). Santiago: Ediciones Universidad Diego Portales.

Lahera, Eugenio (2008). Introducción a las políticas públicas. Santiago: Fondo de Cultura Económica.

Lanzaro, Jorge (2008). “La socialdemocracia criolla”. Nueva Sociedad. № 217, pp. 40-58.

Linz, Juan (1994). "Presidential or Parlamentary Democracy: Does it makes a Difference?", en Linz, Juan y Valenzuela, Arturo (editores). The Failure of Presidential Democracy: Comparative Perspectives, Vol. 1. Baltimore: Johns Hopkins University Press.

Luna, Juan Pablo y Mardones, Rodrigo (2011). “Chile: ¿Se acabaron los partidos?”, Journal of Democracy en español.Vol. 3, pp. 196-212.

Mainwaring, Scott y Shugart MS (compiladores) (2000). Presidencialismo y democracia en América Latina. Buenos Aires: Paidós.

Mainwaring, Scott y Matthew Shugart (editores) (1997). Presidentialism and Democracy in Latinamerica. Cambridge /New York: Cambridge University Press.

Mayol, Alberto. (2012a). El derrumbe del modelo. La crisis de la economía de mercado en el Chile contemporáneo. Santiago de Chile: Ediciones LOM.

Mayol, Alberto. (2012b). No al Lucro. De la crisis del modelo a la nueva era política. Santiago de Chile: Ediciones Debate. 
Muñoz, Oscar y Stefoni, Carolina (Coordinadores) (2003). El período del Presidente Frei Ruiz - Tagle. Santiago: Editorial Universitaria - FLACSO.

Nolte, Detlev (2003). "El Congreso chileno y su aporte a la consolidación democrática en perspectiva comparada". Revista de Ciencia Política.Vol. 23, № 2, pp. 43-67.

North, Douglass (1993). Instituciones, cambio institucional y desempeño económico. México: Fondo de Cultura Económica.

Ominami, Carlos (2011). Secretos de la Concertación. Recuerdos para el futuro. Santiago: La Tercera Ediciones.

Oppliger, Marcel; Guzmán Eugenio (2012). El malestar de Chile: ¿teoría o diagnóstico? Santiago de Chile: RIL editores, Universidad del Desarrollo.

Peters, Guy (2003). El nuevo institucionalismo. Teoría institucional en Ciencia Política. Barcelona: Gedisa Editorial.

Rivera Urrutia, Eugenio (2009). “A medio camino: reformar la reforma previsional del 2008”, Trabajo preparado a solicitud del Senador Carlos Ominami, presidente honorario de la Fundación Chile 21. Inédito.

Salazar, Gabriel (2011). En el nombre del Poder Popular Constituyente (Chile, siglo XXI). Santiago de Chile: Ediciones LOM.

Siavelis, Peter (2000). The president and Congress in postauthoritarian Chile. Pennsylvania: The Pennsylvania State University Press.

Silva, Patricio (2010). En nombre de la razón. Tecnócratas y política en Chile. Santiago: Ediciones Universidad Diego Portales.

Savelis, Peter (2002). "Exaggerated Presidentialism and Moderate Presidents: Excutive Legislative Relations in Chile”, en Morgenstern, Scott y Benito Nacif (Editores). 2002. Legislative Politics in Latin America. Cambridge: Cambridge University Press.

Siavelis, Peter (2006). "Accommodating Informal Institutions and Chilean Democracy”, en Gretchen Helmke, Steven Levitsky (editores). Informal Institutions and Democracy: Lessons from Latin America. Baltimore: Johns Hopkins University Press.

Siavelis, Peter (2009). "Enclaves de la transición y democracia chilena". Revista de Ciencia Política.Vol. 29, No 1, pp. $3-21$.

Varas Augusto (2012 a). La democracia frente al poder. Chile 1990 - 2010. Santiago: Editorial Catalonia.

Varas, Augusto (2012 b). La fronda rediviva. Barómetro de Política y Equidad, N 4, pp. 15-47. 
Viera-Gallo, José Antonio (2012). "El centro de gobierno y su relación con el Congreso", en Fernández Ramil, María de los Angeles y Eugenio Rivera Urrutia (editores), La trastienda del gobierno. El eslabón perdido en la modernización del Estado chileno. Santiago: Editorial Catalonia.

Walker, Ignacio (2003). "Chile: Three Stories of Informal Institutions in a Limited Democracy", ponencia presentada en la conferencia, Informal Institutions and Polítics in Latin America, University of Notre dame, South Bend, ID, Abril 23 - 24.

Entrevistas realizadas:

García, Álvaro, ex Ministro Secretario General de la Presidencia, 10 de septiembre 2012.

Izquierdo, José Miguel, integrante del Segundo Piso (asesoría presidencial), 5 de abril de 2011.

Longueira, Pablo, ex Senador por la Unión Demócrata Independiente y Ministro de Economía, 5 de abril 2011.

Martner, Gonzalo, ex Coordinador Interministerial y ex Subsecretario Subdere, 6 de septiembre 2012.

Ominami, Carlos, ex Ministro de Economía y ex Senador por el PS, fundador del Partido Progresista (PRO), 5 de septiembre 2012.

Vidal, Francisco, ex Ministro Secretario General de Gobierno, ex Ministro del Interior y ex Ministro de Defensa, 4 de septiembre 2012. 